\title{
Use of perioperative ureteral stent in abdominal aortic aneurysm with retroperitoneal fibrosis - A report of two cases -
}

\author{
Junya Kusaka, Shigekiyo Matsumoto, Satoshi Hagiwara, Hironori Koga, and Takayuki Noguchi \\ Department of Anesthesiology and Intensive Care Medicine, Oita University Faculty of Medicine, Oita, Japan
}

Retroperitoneal fibrosis is associated with fibroblast proliferation due to inflammatory changes in adipose/fibrous tissue. Given that aortic dilation in abdominal aortic aneurysm can cause compression of the ureter, abdominal aortic aneurysm complicated by retroperitoneal fibrosis is likely to result in urinary tract obstruction. Accordingly, close attention to changes in perioperative urine volume is warranted when operating on patients with abdominal aortic aneurysm complicated by retroperitoneal fibrosis. We have recently performed laparotomies on two cases of abdominal aortic aneurysm complicated by retroperitoneal fibrosis. In the first case, surgery was performed without the placement of a ureteral stent. The patient developed postrenal acute renal failure caused by postoperative urinary retention. In the second case, ureteral stent placement in advance enabled perioperative management without complications. The clinical course of these cases suggests that, in laparotomy with concomitant retroperitoneal fibrosis, preoperative ureteral stent placement can prevent postoperative complications in the renal and urinary systems. (Korean J Anesthesiol 2012; 63: 76-79)

Key Words: Acute kidney injury, Aortic aneurysm, Retroperitoneal fibrosis, Ureteral stent, Urinary retention.

The causes of most retroperitoneal fibrosis cases are unknown; proliferation of retroperitoneal fibroblasts causes compression of retroperitoneal organs, such as the ureter and kidneys, and occasionally leads to urinary retention [1]. Because the surgical invasiveness of laparotomy may cause edema formation, close attention should be paid to ureteral obstruction during and after surgery. We have recently performed laparotomy on a patient with abdominal aortic aneurysm complicated by retroperitoneal fibrosis, and the patient developed postoperative postrenal acute renal failure (ARF). A few months later, we performed a laparotomy on a different patient with abdominal aortic aneurysm complicated by retroperitoneal fibrosis. In this patient, a ureteral stent was indwelled preoperatively, and the patient had an unremarkable course. These cases suggest the need for preoperative ureteral stent placement when performing laparotomy in patients with

Received: December 30, 2010. Revised: 1st, March 27, 2011; 2nd, June 8, 2011. Accepted: July 31, 2011.

Corresponding author: Satoshi Hagiwara, M.D., Ph.D., Department of Anesthesiology and Intensive Care Medicine, Oita University Faculty of Medicine, 1-1, Idaigaoka-Hasamamachi, Yufu City, Oita 879-5593, Japan. Tel: 81-97-586-5943, Fax: 81-97-586-5949, E-mail: saku@oita-u.ac.jp (c) This is an open-access article distributed under the terms of the Creative Commons Attribution Non-Commercial License (http:// creativecommons.org/licenses/by-nc/3.0/), which permits unrestricted non-commercial use, distribution, and reproduction in any medium, provided the original work is properly cited. 
abdominal aortic aneurysm complicated by retroperitoneal fibrosis.

\section{Case Reports}

\section{Case 1}

The patient was a 78-year-old male with an unremarkable family history; he had a previous history of hypertension and had undergone laparotomies for prostate cancer and colon cancer. On March 21, 2009, the patient complained of severe abdominal pain and visited a nearby clinic. The patient was diagnosed with impending rupture of abdominal aortic aneurysm by abdominal computed tomography (CT) and immediately transferred to our hospital. Careful examination at our hospital revealed that the patient did not have an impending rupture, and thus he underwent elective surgery. Based on subsequent examinations, the patient was diagnosed with inflammatory abdominal aortic aneurysm caused by retroperitoneal fibrosis, and artificial blood vessel replacement was scheduled for April 3.

Preoperative blood test results were found to be within the normal range except for slight deviations for blood coagulation (prothrombin time [PT] 97.2\%, [activated partial thromboplastin time] 56.8\%, Fibrinogen [Fib] $635 \mathrm{mg} / \mathrm{dl}$, antithrombin III [ATIII] $34.4 \%$, D-Dimer $0.88 \mu \mathrm{g} / \mathrm{ml}$ ) and renal function (blood urea nitrogen [BUN] $22.07 \mathrm{mg} / \mathrm{dl}$, Creatinine [Cr] $0.71 \mathrm{mg} / \mathrm{dl}$ ). Preoperative abdominal CT scan revealed that the abdominal aorta measured $31 \mathrm{~mm}$ in its greatest diameter at the point of celiac artery bifurcation, and thickened fibrous tissue was found in the periphery. While the right ureter was functioning

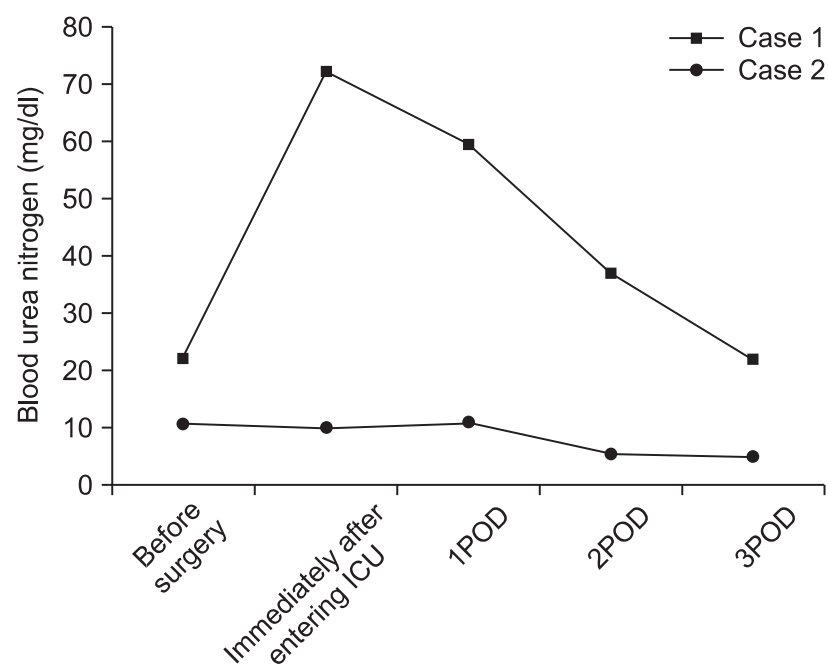

Fig. 1. Changes in perioperative serum blood urea nitrogen. Squares represent changes observed in case 1 ; circles represent changes observed in case 2 . normally, a narrowing of the lumen was seen in the left ureter at the point of renal artery bifurcation where the ureter was enveloped by fibrous tissue surrounding the aorta.

For anesthesia, $100 \mathrm{mg}$ of propofol, $0.25 \mu \mathrm{g} / \mathrm{kg} / \mathrm{min}$ of remifentanil, and $30 \mathrm{mg}$ of rocuronium were used; tracheal intubation was performed. For maintenance of anesthesia, a mixture of $2 \mathrm{~L} / \mathrm{min}$ of oxygen and $3 \mathrm{~L} / \mathrm{min}$ of air, $1-1.5 \%$ sevoflurane, and $0.25-0.5 \mu \mathrm{g} / \mathrm{kg} / \mathrm{min}$ of remifentanil were administered. During surgery, central venous pressure was used as an index for transfusion. Changes in central venous pressure ranged from 7 to $15 \mathrm{mmHg}$, indicating a sufficient volume of infusion. However, anuria continued for two hours from the initiation of surgery. Dopamine was administered, but diuresis was not achieved. Furosemide (40 mg) was tried, but was also ineffective; anuria continued until the end of surgery. Duration of surgery was 8 hours 16 min; duration of anesthesia was 9 hours $52 \mathrm{~min}$. Fibrotic changes in the retroperitoneal cavity were observed during surgery. Accordingly, the patient was diagnosed with retroperitoneal fibrosis. The surgery completed as scheduled, and the intubated patient was transferred to the intensive care unit (ICU).

Fig. 1 and 2 show urine volume and renal function after the patient entered the ICU. After entering the ICU, urine output was approximately $100 \mathrm{ml} / \mathrm{hr}$. A blood test after the patient entered the ICU showed markedly elevated levels of BUN $(72.10 \mathrm{mg} / \mathrm{dl})$ and $\mathrm{Cr}(8.30 \mathrm{mg} / \mathrm{dl})$. Three hours later, the blood test was repeated and showed further elevated levels of BUN (70.35 mg/dl) and $\mathrm{Cr}(8.58 \mathrm{mg} / \mathrm{dl})$. Abdominal ultrasonography was performed to examine the cause; significant bilateral hydronephrosis was observed (Fig. 3). Furthermore, bilateral ureteral stenosis was observed by retrograde pyelography.

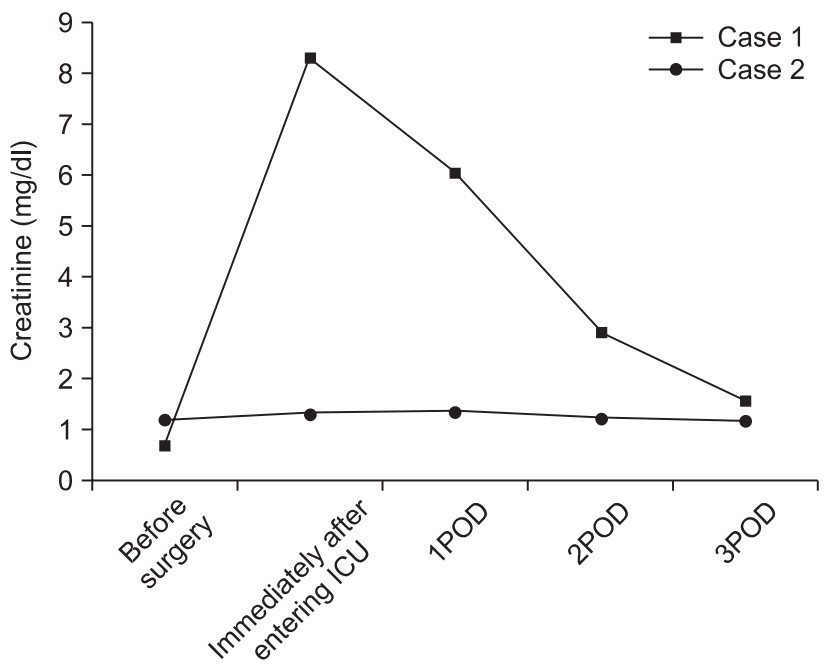

Fig. 2. Changes in perioperative serum creatinine. Squares represent changes observed in case 1 ; circles represent changes observed in case 2 . 


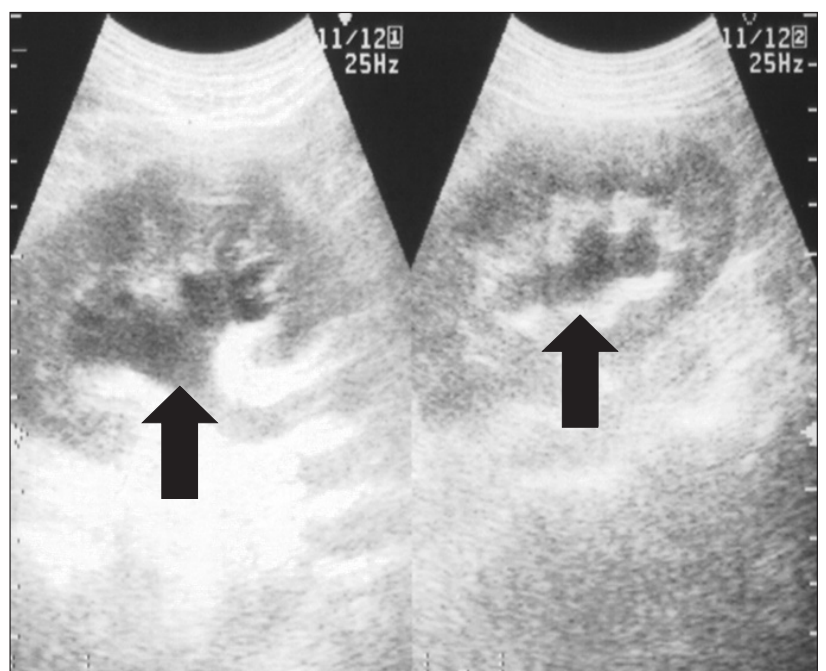

Fig. 3. Image captured by abdominal ultrasonography in case 1 . Arrows are directed to the enlarged renal pelvis.

The patient was diagnosed with postrenal ARF and a bilateral ureteral stent was placed. During placement, ureters on both sides exhibited strong resistance at insertion. In particular, severe stenosis of the left ureter was observed at the L4-5 level. Diuresis was satisfactory after ureteral stent placement (Fig. 4). Renal function swiftly improved, and there was no need for blood purification therapy. By postoperative day 10, the patient's renal function returned to preoperative levels (BUN $19.7 \mathrm{mg} / \mathrm{dl}, \mathrm{Cr} 0.79 \mathrm{mg} / \mathrm{dl}$ ).

\section{Case 2}

The patient was a 41-year-old male with unremarkable family history; he had a history of hypertension since 2006 and had undergone an aortic arch replacement for acute aortic dissection in September 2006.

Prior to aortic arch replacement, the patient had an abdominal CT scan which revealed abdominal aortic aneurysm and bilateral iliac artery aneurysm. The patient was admitted to the hospital for treatment of abdominal aortic aneurysm. Preoperative CT suggested retroperitoneal fibrosis.

Preoperative blood tests were unremarkable with the exception of slightly elevated Fib (635 mg/dl). Preoperative imaging findings revealed abdominal aortic aneurysm at the renal artery level, with $42 \mathrm{~mm}$ as the greatest diameter. Furthermore, an aneurysm measuring $43 \mathrm{~mm}$ in its greatest diameter was observed in a region from the lower abdominal aorta at the renal artery bifurcation to the bilateral iliac artery. Right hydronephrosis and left ureteropelvic duplication were also observed. The urinary tract of this patient was joined together halfway (incomplete duplication). A ureteral stent had

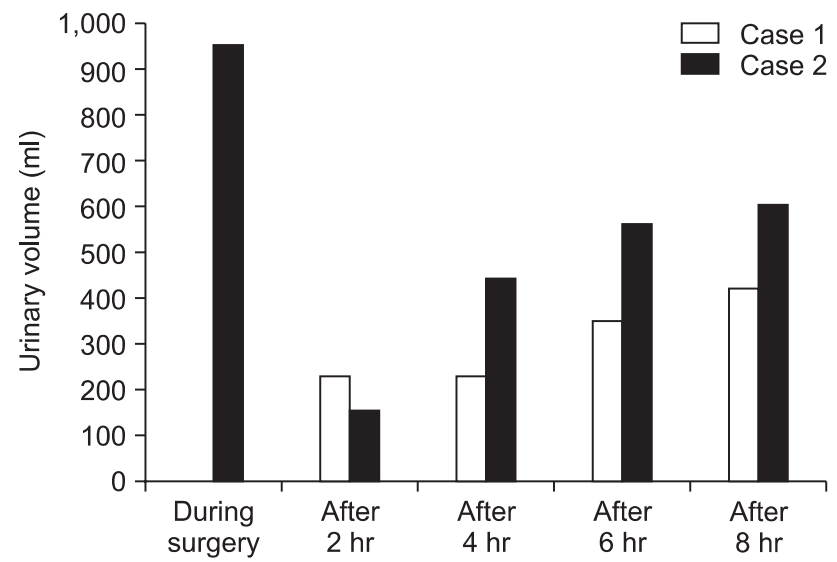

Fig. 4. Changes in perioperative urine volume. White bars represent changes observed in case 1 ; black bars represent changes observed in case 2 .

already been indwelled for left ureteropelvic duplication.

The day before surgery, an epidural catheter was indwelled at Th 11/12. For anesthesia, $150 \mathrm{mg}$ of propofol, $0.25 \mu \mathrm{g} / \mathrm{kg} / \mathrm{min}$ of remifentanil, and $10 \mathrm{mg}$ of vecuronium were used; tracheal intubation was performed. For maintenance of anesthesia, a mixture of $2 \mathrm{~L} / \mathrm{min}$ of oxygen and $3 \mathrm{~L} / \mathrm{min}$ of air, $1-1.5 \%$ of sevoflurane, and $5 \mathrm{ml}$ of $1 \%$ mepivacaine were intermittently administered. Central venous pressure was used as an index for transfusion. Changes in central venous pressure ranged from 7 to $12 \mathrm{mmHg}$, indicating a sufficient volume of infusion. Perioperative urine volume was satisfactory $(950 \mathrm{ml})$ at a rate of $30-300 \mathrm{ml} / \mathrm{h}$. Fibrotic changes were observed in the retroperitoneal cavity during surgery. Accordingly, the patient was diagnosed with retroperitoneal fibrosis. Duration of surgery was 8 hours $16 \mathrm{~min}$; duration of anesthesia was 9 hours $52 \mathrm{~min}$. The surgery completed as scheduled, and the intubated patient was transferred to the ICU. In contrast to case 1, urine output was satisfactory after entering the ICU. The patient exhibited no marked deterioration in renal function during the perioperative period and had a satisfactory outcome.

\section{Discussion}

In surgery for abdominal aortic aneurysm, decreased renal blood flow caused by aortic blockage is associated with a high risk of postoperative renal dysfunction. However, if renal function is normal prior to surgery, and appropriate perioperative management is conducted, postoperative postrenal ARF rarely occurs. In general, the most common cause of ARF is acute tubular necrosis, while postrenal-related causes account for approximately $13 \%$ of the total [2].

In case 1 , the patient developed postoperative postrenal ARF. Possible causes included: the presence of concomitant 
retroperitoneal fibrosis; narrowing of renal tubules caused by retroperitoneal fibrosis; and edema resulting from laparotomy, which led to ureteral compression that progressed during surgery. Postrenal failure was promptly diagnosed by measures such as examining perioperative urine volume, renal function by blood sampling, and presence or absence of hydronephrosis by abdominal ultrasonography. These measures enabled the patient to receive appropriate treatment before his condition became serious and allowed for his steady recovery.

In case 2 , a ureteral stent was preoperatively indwelled in the left ureter. Throughout the perioperative period, the patient had satisfactory urine output with no sign of deterioration in renal function or complications of the urinary tract. Consequently, the patient had a better outcome than the patient in case 1 .

Retroperitoneal fibrosis, a relatively rare disease in Japan, forms adjacent to retroperitoneal organs and, in some cases, can occlude the ureter, which may cause urinary retention. While retroperitoneal fibrosis is caused by various factors, idiopathic retroperitoneal fibrosis with unknown causes account for a majority of cases (70\%). Other causes include: malignant tumor, infection, retroperitoneal injury, autoimmunity, and history of laparotomies [3]. This syndrome is now classified in the inflammatory pathology group, which includes chronic periaortitis and inflammatory aneurysm, all of which will lead to fibrosis [4]. In patients with inflammatory abdominal aortic aneurysm, the possibility of complications with retroperitoneal fibrosis should be considered [5]. In case 1, the patient was diagnosed with inflammatory abdominal aortic aneurysm. This suggests that in some patients, abdominal aortic aneurysm may be complicated by retroperitoneal fibrosis. Therefore, when performing emergency aortic surgery on patients who exhibit urinary retention, retroperitoneal fibrosis should be considered a disease that requires differential diagnosis.

Magnetic resonance imaging (MRI) is an effective method for diagnosing retroperitoneal fibrosis. Steroid therapy is a major treatment that is effective during inflammation. Surgical resection may be considered in cases where patients develop marked fibrosis or have complications such as postrenal failure with ureteral stenosis or inflammatory abdominal aortic aneurysm. One study reports that in inflammatory abdominal aortic aneurysm, proliferation of peripheral fibrous tissue eventually envelops the ureter, causing it to become narrower [6]. Although abdominal aortic aneurysm is rarely a cause of ureteral stenosis, one study reports that unilateral or bilateral ureteral stenosis was observed in $62 \%$ of abdominal aortic aneurysm cases [7]. Another study on inflammatory abdominal aortic aneurysm caused by retroperitoneal fibrosis reports that new hydronephrosis occurred after surgery in $19 \%$ of cases [8]. Therefore, there is a high likelihood that bilateral ureteral stenosis occurs after surgery. Given that ureteral occlusion tends to occur during the perioperative period, intraoperative management is important; during laparotomy on patients with concomitant retroperitoneal fibrosis, we should bear in mind that ureteral stenosis may occur due to ureteral edema or edema formed in adjacent tissue as a result of surgical invasion. To avoid such problems, it may be worth considering the use of steroid and albumin/fresh frozen plasma. Furthermore, as seen in the second case, preoperative ureteral stent placement may allow for safer management.

In abdominal surgery on such patients, ureteral occlusion may occur during the perioperative period, even when the patient's renal function was normal before surgery. Therefore, preoperative ureteral stent placement was considered necessary. Furthermore, for early detection of postoperative hydronephrosis, it is important to measure urine volume, examine renal function by blood sampling, and determine the presence or absence of hydronephrosis by abdominal ultrasonography. If there is suspicion of postrenal failure, retrograde pyelography should be performed, and ureteral stent placement is required immediately after definitive diagnosis.

\section{References}

1. Cronin CG, Lohan DG, Blake MA, Roche C, McCarthy P, Murphy JM. Retroperitoneal fibrosis: a review of clinical features and imaging findings. AJR Am J Roentgenol 2008; 191: 423-31.

2. Liaño F, Pascual J. Epidemiology of acute renal failure: a prospective, multicenter, community-based study. Madrid Acute Renal Failure Study Group. Kidney Int 1996; 50: 811-8.

3. Koep L, Zuidema GD. The clinical significance of retroperitoneal fibrosis. Surgery 1977; 81: 250-7.

4. Parums DV. The spectrum of chronic periaortitis. Histopathology 1990; 16: 423-31.

5. Jagadesham VP, Scott DJ, Carding SR. Abdominal aortic aneurysms: an autoimmune disease? Trends Mol Med 2008; 14: 522-9.

6. Vaglio A, Salvarani C, Buzio C. Retroperitoneal fibrosis. Lancet 2006; 367: 241-51.

7. Scheel PJ Jr, Feeley N. Retroperitoneal fibrosis: the clinical, laboratory, and radiographic presentation. Medicine (Baltimore) 2009; 88: 202-7.

8. von Fritschen U, Malzfeld E, Clasen A, Kortmann H. Inflammatory abdominal aortic aneurysm: A postoperative course of retroperitoneal fibrosis. J Vasc Surg 1999; 30: 1090-8. 